\title{
Near-complete phase transfer of single-wall carbon nanotubes by covalent functionalization ${ }^{\dagger}$
}

\author{
BHALCHANDRA KAKADE, SANJAY PATIL, BHASKAR SATHE, \\ SURESH GOKHALE and VIJAYAMOHANAN PILLAI* \\ Physical and Materials Chemistry Division, National Chemical Laboratory, Dr. Homi Bhabha Road, \\ Pune 411008 \\ e-mail: vk.pillai@ncl.res.in
}

\begin{abstract}
We describe here an efficient phase transfer of single wall carbon nanotubes (SWNTs) from aqueous to non-aqueous media using a unique amide functionalization route, where water soluble SWNTs $(2.6 \mathrm{mg} / \mathrm{mL})$ are effectively transferred to solvents like chloroform, toluene and $\mathrm{CS}_{2}$. A maximum of $30 \mathrm{wt} \%$ of oxygenated groups have been generated on the side walls by rapid microwave treatment, leading to a solubility of more than $2.6 \mathrm{mg} / \mathrm{mL}$ in water. Approximate surface amine coverage of $50 \%$ has been accomplished after oxalyl chloride treatment as inferred from thermogravimetry and X-ray photoelectron spectroscopy by controlling several key parameters associated with the extent of functionalization including purity of the sample, temperature and time.
\end{abstract}

Keywords. Carbon nanotubes; nanostructures; microscopy; microwave; solubility of SWNTs.

\section{Introduction}

Recently, functionalization of single-walled carbon nanotubes has attracted great interest in numerous areas of research, ranging from high-strength polymer nanocomposites, ${ }^{1,2}$ optics ${ }^{3}$ and bio-technology $y^{4,5}$ to energy storage. This is mainly because, in order to take the full advantage of potential applications of CNTs, the inert surface of nanotubes with intrinsic van der Waals forces must be overcome by linking them to appropriate molecules, which in turn, can make them more adaptable/soluble in various solvents. More significantly, diazotization of SWNTs enables the separation of metallic SWNTs from semiconducting type as illustrated by Smalley and co-workers. ${ }^{6}$ Also, several methods have been subsequently attempted recently to functionalize SWNTs, ${ }^{7-17}$ with the primary objectives of tuning their electronic properties for exploiting their potential applications along with alleviating common processing obstacles like bundling or flocculation while using the pristine form. More recent studies undoubtedly reveal that, the solubility of SWNTs in water can be enhanced along with efficient exfoliation of nanotube bundles due to special methods like superacid treatment, ${ }^{18}$ attachment of arylsulphonated

\footnotetext{
${ }^{\dagger}$ Dedicated to Prof. C N R Rao on his 75th birthday

*For correspondence
}

groups, ${ }^{19}$ and microwave treatment in presence of oleum and nitric acid at elevated pressures. ${ }^{20}$ However, several issues regarding the extent of functionalization and contamination affecting their purity have not been adequately analysed in these cases. For example, it is not clear how the low extent of $-\mathrm{COOH}$ group attachment (less than $5 \mathrm{wt} \%$ of oxygenated side groups) on the sidewalls/ends, generates such a highly unusual amount of amine functionalization (up to $70 \mathrm{wt} \%$ ). ${ }^{21-23}$ Whether the present methods of acid functionalization of CNTs are sufficient or not to produce adequate amount of $-\mathrm{COOH}$ groups for further efficient derivatization is not clear to date. More significantly, the purification of SWNTs-COCl by repeated centrifugation and washing with solvents could deactivate (due to moisture sensitive $-\mathrm{COCl}$ groups) the acyl moieties, which is not well explained in all these reports. Hence, there is an immediate need to explore alternative synthetic procedures in order to achieve greater efficiency and high purity using covalent functionalization strategies due to the pre-eminent role in all application of SWNTs.

Here, we report such a new functionalization strategy by a simple covalent amide formation of SWNTs with tridecylamine through oxalyl chloride treatment, which enables a higher, yet quantitative, degree of functionalization facilitating subsequent complete phase transfer from aqueous to a non- 
aqueous media. The use of oxalyl chloride gives remarkable purity in the final product along with easy processing characteristics due to its lower boiling point, whereas, thionyl chloride ${ }^{24}$ or dicyclohexylcarbodiimide $^{13}$ requires many rigorous purification steps to remove excess reagents or unwanted byproducts.

\section{Experimental}

\subsection{Preparation of water soluble SWNTs}

In a typical experiment, $50 \mathrm{mg}$ of SWNT soot obtained from Aldrich (purity: $>60 \%$; diameter: 0.7$1.3 \mathrm{~nm}$; synthesized by CVD method) was taken in a teflon cylinder with a grooved stopper containing a $1: 1$ mixture of $98 \% \mathrm{H}_{2} \mathrm{SO}_{4}$ and $70 \% \mathrm{HNO}_{3}$. This was subjected to microwave (MW) irradiation in a domestic oven for 4 min (separated by $60 \mathrm{~s}$ off-time interval) using $60 \%$ of its total power of $700 \mathrm{~W}$. The rigorous conditions imposed by the MW render rapid breaking of graphitic $\mathrm{C}=\mathrm{C}$ to develop large amount of $-\mathrm{OH},-\mathrm{COO}^{-}$and $-\mathrm{SO}_{3} \mathrm{H}$ groups on the sidewalls of the SWNTs. Soluble residue was first filtered through a $0.2 \mu \mathrm{m}$ PTFE membrane and separated out from the unfiltered carbon matrix. The nanotubes passed through the membrane were collected separately after their thorough purification by dialysis to yield shorter analogues. (Microwave treatment to 'as-received SWNT' sample would further add to the disordered carbon mass. However, most of this nontubular carbon and catalysts have been removed during the first filtration and SWNTs deposited on the membrane has been used for further covalent functionalization). The unfiltered residue was again dispersed in $3 \mathrm{M} \mathrm{HCl}$ and sonicated for $30 \mathrm{~min}$ to develop $-\mathrm{COOH}$ groups rather than $\mathrm{COO}^{-}$, which perhaps could hinder further functionalization. Acidified nanotubes were subjected to dialysis for 2 days to remove excess of acid content (until the $\mathrm{pH}$ of the water becomes neutral). SWNTs obtained through such MW treatment show a remarkable solubility of $2.6 \mathrm{mg} / \mathrm{mL}$, whereas, this value has been enhanced in the case of SWNTs in the filtrate $(3-4 \mathrm{mg} / \mathrm{mL})$ without any serious damage on the sidewalls (as judged by Raman Spectroscopy and TEM). An irradiation time of $4 \mathrm{~min}$ is systematically chosen in the present study in order to achieve better sidewall functionalization as well as to restrict further damaging/cutting of nanotubes that might lead to loss in weight.

\subsection{Amine functionalization of water soluble SWNTs}

About $40 \mathrm{mg}$ of water-soluble SWNTs (s-SWNTs) having $2.6 \mathrm{mg} / \mathrm{mL}$ was ultrasonicated for $10 \mathrm{~min}$ in presence of $10 \mathrm{~mL}$ of oxalyl chloride, (taking extreme care during handling of oxalyl chloride) and the reaction was carried out in an argon atmosphere. The mixture was then refluxed for $24 \mathrm{~h}$ at $60^{\circ} \mathrm{C}$ to obtain a higher degree of acylchloride functionalization (SWNTs-COCl); excess of oxalyl chloride was removed under vacuum using rota-evaporation. SWNTs-COCl was then mixed with $1 \mathrm{~g}$ solid tridecylamine (TDA; $\mathrm{CH}_{3}\left(\mathrm{CH}_{2}\right)_{12} \mathrm{NH}_{2}$ ) and refluxed at $40^{\circ} \mathrm{C}$ (m.p. of TDA: $\sim 30^{\circ} \mathrm{C}$ ) for $40 \mathrm{~h}$. Excess of TDA was then removed by washing with copious amount of THF with successive sonication and centrifugation. The resultant SWNTs modified by TDA (37 mg) were found to be highly soluble in $\mathrm{CHCl}_{3}$ and $\mathrm{CS}_{2}$, as revealed in scheme 1. All samples were characterized by UV-Vis absorption spectroscopy, Diffused reflectance infrared spectroscopy (DRIFT), Proton nuclear magnetic resonance ( ${ }^{1} \mathrm{H}$ NMR), X-ray photoelectron spectroscopy (XPS), Thermogravimetry (TG) and Raman spectroscopy.

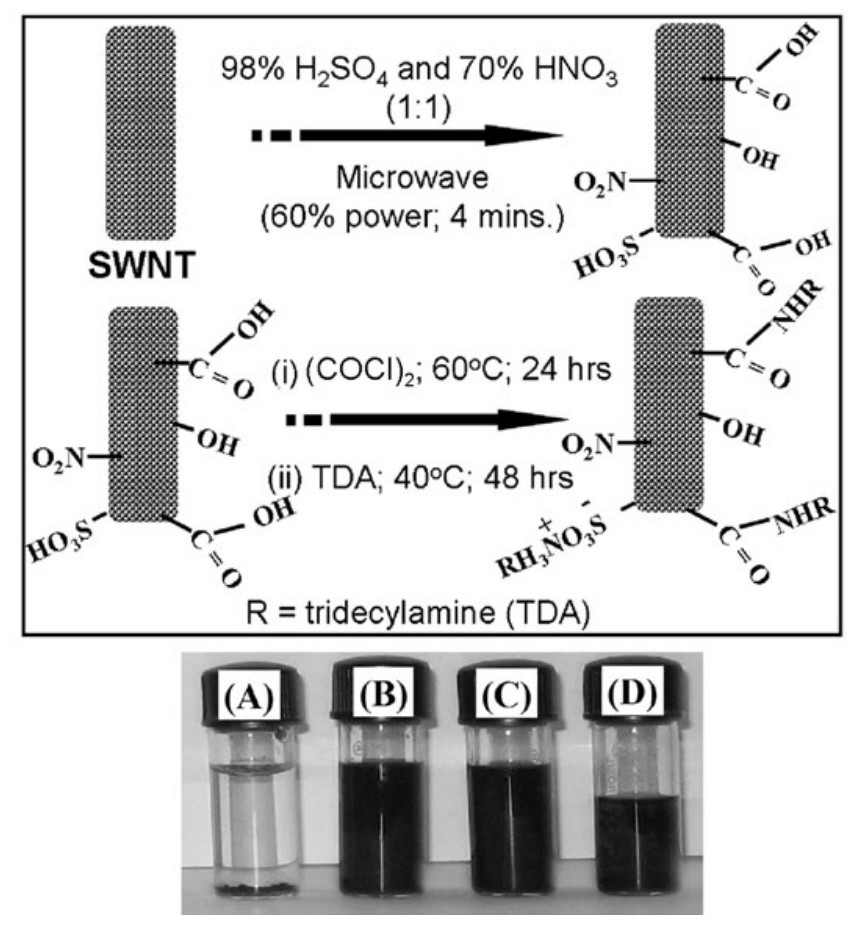

Scheme 1. Schematic depiction of the formation of water soluble SWNTs (s-SWNTs) using microwave treatment followed by tridecylamine functionalization via oxalyl chloride; photographs reveal (A) pristine SWNTs, (B) s-SWNTs (in water; $2.6 \mathrm{mg} / \mathrm{mL}$ ) and after amide formation SWNTs, (C) in $\mathrm{CHCl}_{3}$, (D) in $\mathrm{CS}_{2}$. 


\subsection{Materials characterization}

The morphology of all these SWNT samples was examined using a JEOL JEM 1200EX transmission electron microscope (TEM) operated at an accelerating voltage of $120 \mathrm{kV}$. Changes in the density of states, after functionalization, were monitored by Varian model Cary 50 Dual Beam UV-Vis spectrophotometer. The pristine SWNT sample was ultrasonicated in millimolar sodium dodecylsulphate (SDS) in water to get a good dispersion of SWNTs, whereas the SWNTs-CONH $\left(\mathrm{CH}_{2}\right)_{12} \mathrm{CH}_{3}$ sample was dissolved in toluene and UV-Vis spectra were recorded. Perkin Elmer Instruments-Spectrum One FTIR spectrometer was used for functional group analysis in DRIFT mode. DRIFT analysis was performed after thorough mixing of small amount of sample in $\mathrm{KBr}$ followed by drying in desiccator. TG analysis was carried out on a TGA Q5000V2.4 build 2-3 thermal analyzer by heating about $2 \mathrm{mg}$ of carbon sample from $50^{\circ} \mathrm{C}$ to $900^{\circ} \mathrm{C}$ at a rate of $10^{\circ} \mathrm{C} /$ min in air. In order to understand the surface functionality present on water-soluble and TDA functionalized SWNTs samples, proton NMR ( ${ }^{1} \mathrm{H}$ NMR) studies have been performed on a Bruker MSL $400 \mathrm{MHz}$ instrument using DMSO (d6) as a dispersing solvent to detect magnetically different protons after functionalization. X-ray photoelectron spectroscopic (XPS) measurements were carried out on a VG Micro Tech ESCA 3000 instrument at a pressure of $>1 \times 10^{-9}$ Torr (pass energy of $50 \mathrm{eV}$ with an electron take off angle $60^{\circ}$ and an overall resolution of $1 \mathrm{eV}$ ) using $\mathrm{MgK}_{\alpha}$ radiation $(1253.6 \mathrm{eV})$. The binding energy of Cls peak was fixed to $284.5 \mathrm{eV}$ and all other peaks were calibrated with reference to this graphitic $\mathrm{Cls}$ peak. The background was subtracted by Shirley method. Raman analysis was performed on a JASCO confocal Raman spectrometer using $532 \mathrm{~nm}$ green laser (NRS $1500 \mathrm{~W}$ ) in order to obtain the effect of functionalization.

\section{Results and discussion}

Figure 1 indicates high resolution TEM images of pristine-SWNTs, s-SWNTs and SWNTs-CONH $\left(\mathrm{CH}_{2}\right)_{12} \mathrm{CH}_{3}$, showing copious sites of defects after the microwave processing and amine functionalization. This unresolved image (but still graphitic fringes are seen) of $s$-SWNTs also indicates some electrostatic interactions among nanotube bundles. In fact, an effective debundling would lead to the enhancement in the solubility, although the bundles are still clearly seen in figure 1(b). This could be clarified by the Fast Fourier Transform (FFT) image of the figure 1(b), shown in the inset, where the strong 002 reflection reveals the presence of graphitic interactions (intertubular). Amine functionalized SWNTs show a large amount of wrapping of organic molecules around nanotubes (as shown in figure $1(\mathrm{c}-\mathrm{d})$, thus precluding high resolution images, which reveal a higher extent of functionalization.

A comparison of absorption spectra in figure $2 \mathrm{a}$ clearly shows the disappearance of the van Hove singularities after MW treatment and amine functionalization, indicating the breaking of electronic structure due to the generation of defect sites on the sidewalls. In pristine SWNT sample, the presence of mixture of both semiconducting and metallic SWNTs shows individual absorption peaks that allows for monitoring of valence electrons. Further, the functionalization with tridecylamine gives localization of these valence electrons resulting into decay of such maxima in the spectrum.

DRIFT spectra (figure 2b) of all samples, including pristine SWNTs, s-SWNTs and SWNTs-CONH $\left(\mathrm{CH}_{2}\right)_{12} \mathrm{CH}_{3}$ show a peak at $1580 \mathrm{~cm}^{-1}$ corresponding to the active carbon stretching mode of the nanotubes. A new peak at $1600 \mathrm{~cm}^{-1}$ in case of s-SWNTs is attributed to the -COO-groups, which facilitates the solubility of SWNTs preventing further amide bonding. Alkyl groups exhibit absorptions at 2850 and $2922 \mathrm{~cm}^{-1}$ respectively due to typical $-\mathrm{C}-\mathrm{H}$ stretching frequencies. Also a sharp peak at $1650 \mathrm{~cm}^{-1}$ has been observed corresponding to a stretching frequency of carbonyl $(v \mathrm{C}=0)$ due to amide formation.

${ }^{1} \mathrm{H}$ NMR $\left(400 \mathrm{MHz}\right.$, DMSO- $\left.d_{6}\right)$ of SWNTs$\mathrm{CONH}\left(\mathrm{CH}_{2}\right)_{12} \mathrm{CH}_{3}$ shows the presence of long aliphatic chain on the sidewalls as well as on the ends of SWNTs: chemical shift $\delta 0.89\left(\mathrm{CH}_{3}\right), 1.33\left(\mathrm{CH}_{2}\right)$. The $-\mathrm{CH}_{2}$ group directly attached to the amide group at the ends of the SWNTs shows several broad signals in the range of $\delta 2.8$ to 3.2 ; the integral of all of these signals corresponds to $2 \mathrm{H}$, which suggests the existence of magnetically different types of SWNTs in our samples. In addition, the signals ranging from $\delta, 6.94$ to 7.20 indicate the development of few $-\mathrm{C}-$ $\mathrm{H}$ bonds on benzene rings (aromatic $\mathrm{H}$ ) of SWNTs, creating wall defects. Also, the presence of a peak at $\delta 7.71$ indicates amido proton, although the weak intensity could contradict subsequent description of large amidation (figure 3). The protons in the SWNTs-CONH $\left(\mathrm{CH}_{2}\right)_{12} \mathrm{CH}_{3}$, in which the tride- 


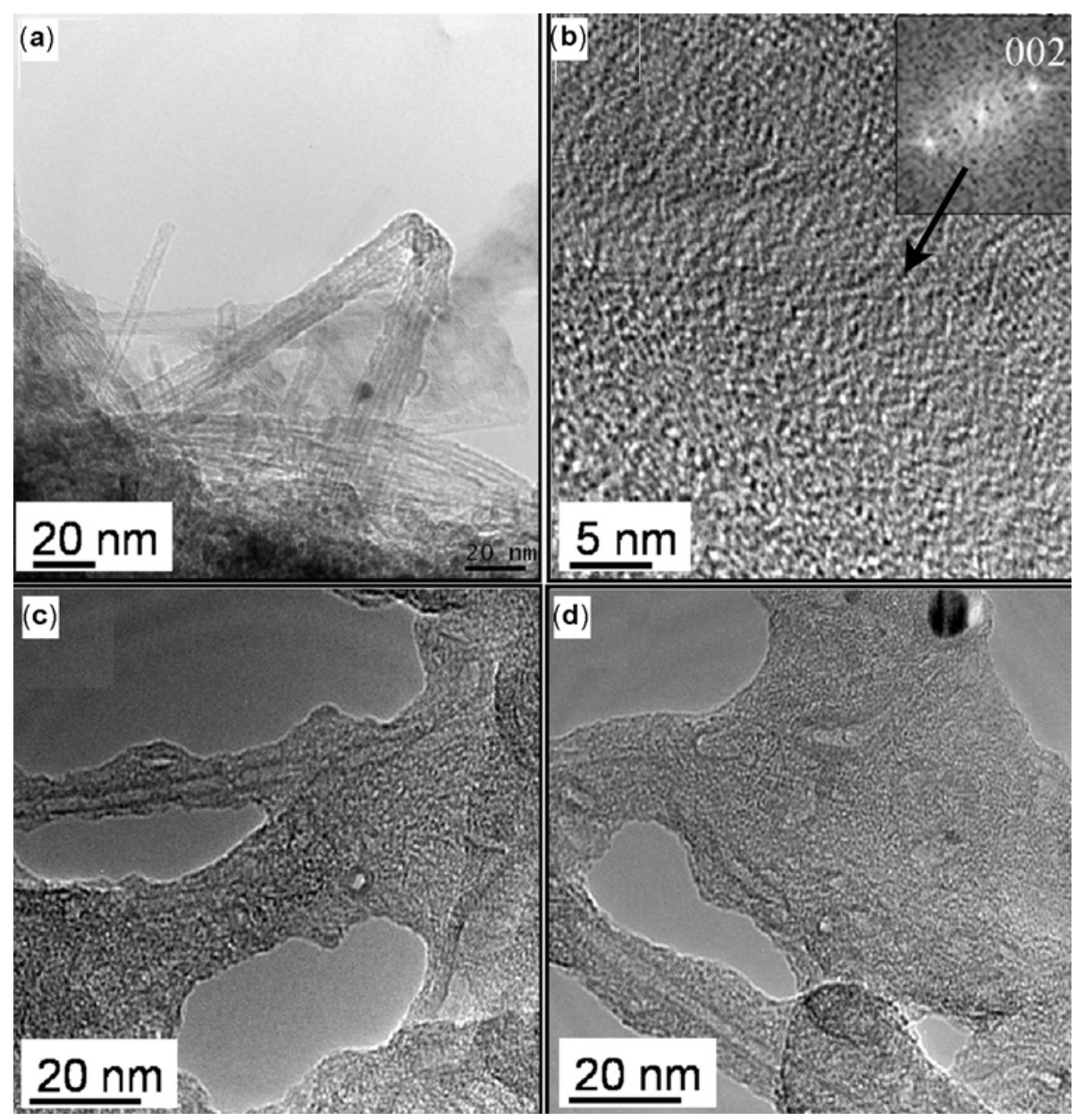

Figure 1. High resolution TEM images of (a) pristine SWNTs, (b) s-SWNTs and (c-d) SWNTs-CONH $\left(\mathrm{CH}_{2}\right)_{12} \mathrm{CH}_{3}$, which clearly show morphological differences after functionalization; s-SWNTs show the debundling to certain extent, while the 002 reflection in Fast Fourier Transform (FFT) image in the inset of (b) clearly shows strong van der Waal's interaction among individual nanotubes; amine functionalized SWNTs show a large amount of organic molecules around nanotubes, hence precluding resolved images of the nanotubes.

(a)

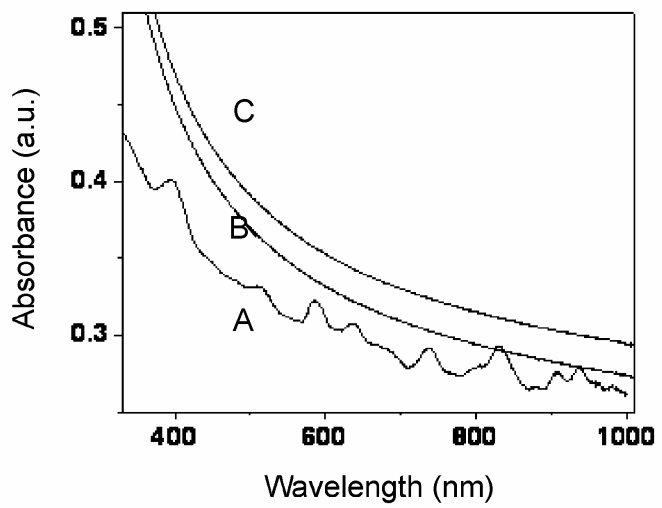

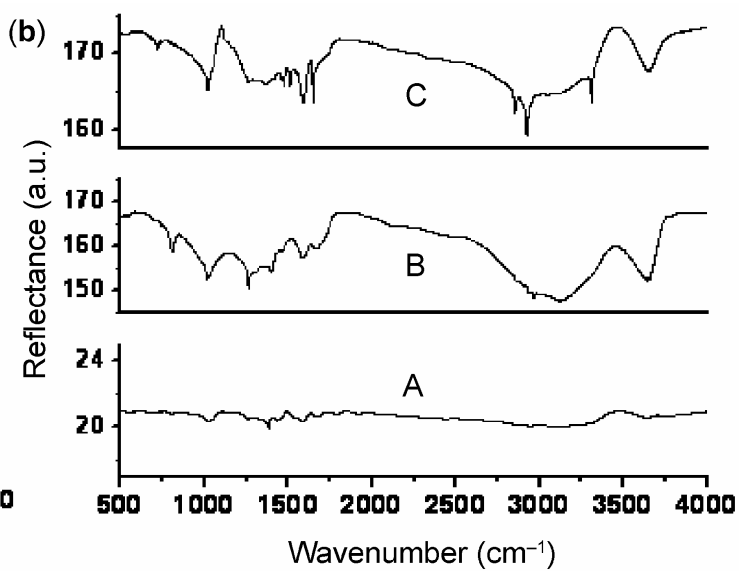

Figure 2. (a) UV-Vis absorption spectra of (A) pristine SWNTs dispersed in millimolar SDS solution in water, (B) s-SWNTs, (C) SWNTs- $\mathrm{CONH}\left(\mathrm{CH}_{2}\right)_{12} \mathrm{CH}_{3}$ in toluene, which shows a complete loss of structure (van Hove singularities) upon chemical treatments, revealing the formation of defects due to conversion of $s p^{2}$ to $s p^{3}$ carbon. (b) Superimposed DRIFT spectrum of (A) pristine SWNTs, (B) s-SWNTs and (C) SWNTs-CONH $\left(\mathrm{CH}_{2}\right)_{12} \mathrm{CH}_{3}$ performed using $\mathrm{KBr}$. 
cylamine is covalently attached to the SWNTs, are much more affected by the backbone of SWNTs network, and it is the strong amidation that is responsible for the drastic changes in the ${ }^{1} \mathrm{H}$ NMR spectrum of the tridecylamine. This also indicates that, there is no free tridecylamine present in the sample.

In addition, more than $50 \%$ of the TDA attached to SWNTs is confirmed by TG analysis. A single inflection in the weight loss (figure 4B) pattern up to $300^{\circ} \mathrm{C}$ indicates the presence of only covalently

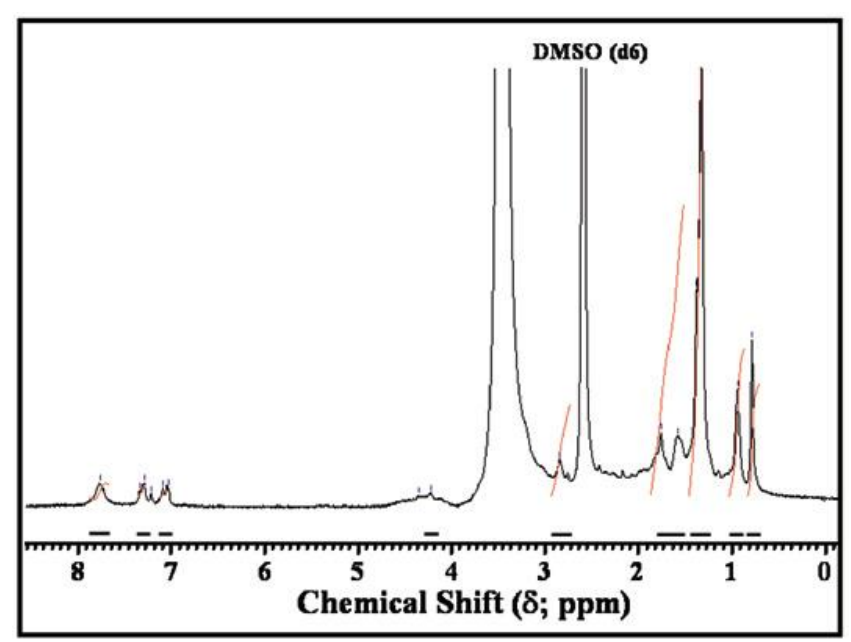

Figure 3. ${ }^{1} \mathrm{H}$ NMR spectrum of SWNTs- CONH $\left(\mathrm{CH}_{2}\right)_{12} \mathrm{CH}_{3}$ performed in DMSO- $d_{6}$ at $400 \mathrm{MHz}$.

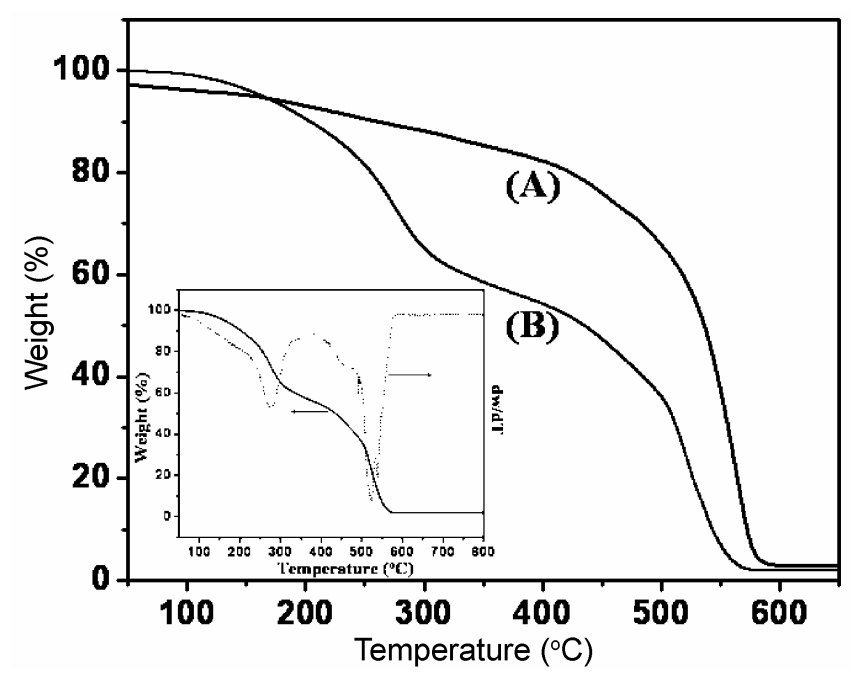

Figure 4. TG analysis of (A) s-SWNTs and (B) SWNTs-CONH $\left(\mathrm{CH}_{2}\right)_{12} \mathrm{CH}_{3}$ performed in dry oxygen, showing $50 \%$ loss in weight that implies a higher degree of amidation on defect walls. Inset shows a DT curve for SWNTs-CONH $\left(\mathrm{CH}_{2}\right)_{12} \mathrm{CH}_{3}$, clearly indicating two step weight loss. bonded TDA, revealing the high purity. One of the key reasons in the enhancement of such a large amidation might be the abundance $(\sim 8 \mathrm{wt} \%)$ of carboxylic groups generated during the MW treatment and consequently a considerably higher degree of acylation with oxalyl chloride. Inset of figure 3 shows a differential thermal analysis curve for SWNTs$\mathrm{CONH}\left(\mathrm{CH}_{2}\right)_{12} \mathrm{CH}_{3}$ that gives a smaller hump at $460^{\circ} \mathrm{C}$ (corresponding to other stronger functional moieties on the side walls) in addition to two sharp inflexions, revealing a clear evidence for TDA functionalization. Also, it is consistent with the fact that, other reactive sites like $-\mathrm{SO}_{3} \mathrm{H}$ are produced during the oxidative treatments along the tubular walls and at the ends. The volume of the SWNTs is also found to expand (approximately 30\%) after the functionalization due to the exfoliation of nanotube bundles forming shorter analogues.

Structural variations are also clarified by the XPS analysis (figure 5), where a reduced peak corresponding $\mathrm{Cls}$ core shells directly indicates a remarkable extent of functionalization. The presence of other surface functionalities on the sidewalls is also confirmed by XPS analysis especially when the results are compared with those for pristine.

In addition, using relative amounts of $\mathrm{C}, \mathrm{N}$ and $\mathrm{S}$ present in the sample and normalizing to the $S$, we find that there are $16 \mathrm{~N}$ atoms (from amide bonds) per $1 \mathrm{~S}$ atom (from sulphonate). This means that approximately $7 \mathrm{wt} \%$ of $\mathrm{N}$ and $1 \mathrm{wt} \%$ of $\mathrm{S}$ is present on the side walls after TDA amidation, which is in good agreement with the results of the TG analysis. Accordingly, figure 4a shows the XP spectrum of sSWNTs where, the C1s spectrum has been deconvoluted to five peaks; a major peak at $284.4 \mathrm{eV}$ is assigned to graphitic $\mathrm{C}-\mathrm{C}$ bonds, while four minor contributions at $285.6,286.6,288.5$ and $289.5 \mathrm{eV}$ could be attributed to the carbon atoms attached to other atoms in four different environments such as, $\mathrm{OH}, \mathrm{NO}_{2}, \mathrm{COOH}$ and $\mathrm{SO}_{3} \mathrm{H}$ respectively. Except a major (74092; after background subtraction) peak at $284.4 \mathrm{eV}$ corresponding to graphitic $\mathrm{C}-\mathrm{C}$ bond, a relatively intense (20868: after background subtraction, FWHM: 2.19) peak at $288.5 \mathrm{eV}$ is observed due to $\mathrm{COOH}$ groups, which are utilized for the formation of $-\mathrm{CONH}$ functionalization with TDA. By normalizing with respect to oxygen the composition of oxygen on the side wall after MW treatment is found to be $30 \mathrm{wt} \%(\sim 4.3 \mathrm{C}$ atoms per $1 \mathrm{O}$ atom), which is the maximum value as compared to the previous reports. However, five different oxygen functionalities are observed corre- 

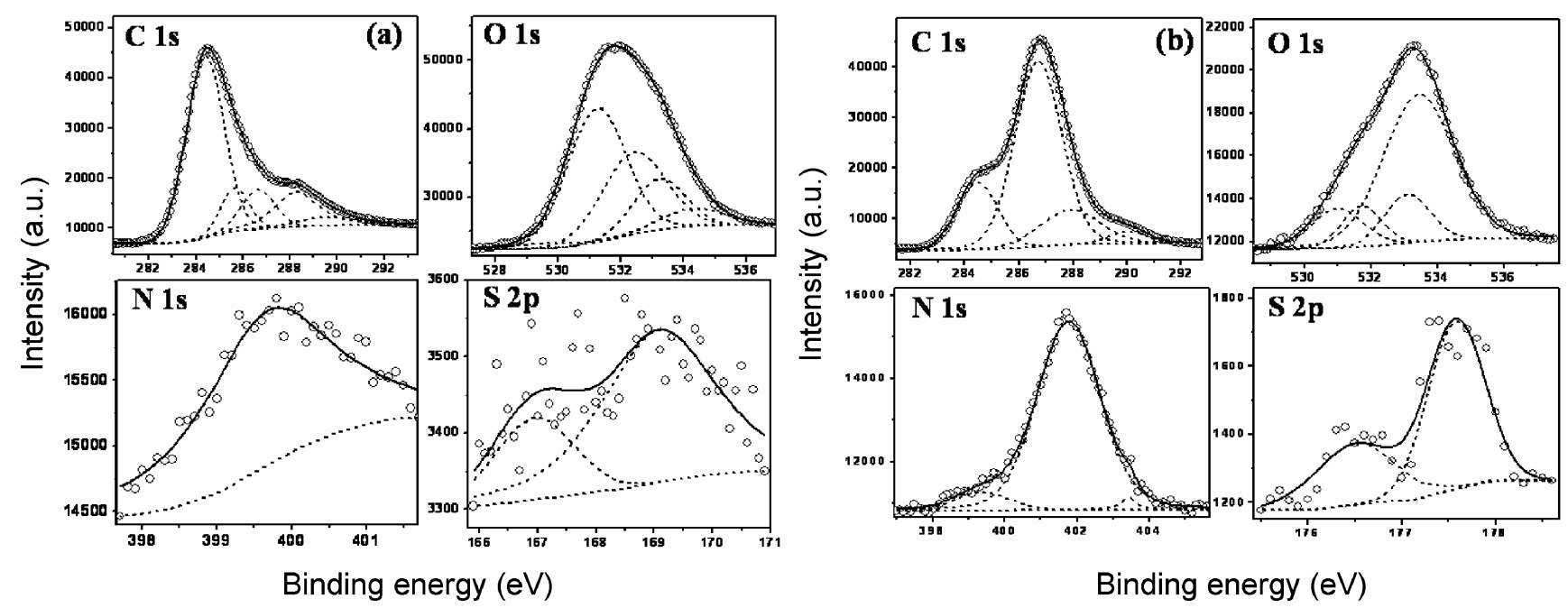

Figure 5. XP spectra of (a) s-SWNTs and (b) SWNTs-CONH(CH $\left(\mathrm{CH}_{2}\right)_{12} \mathrm{CH}_{3}$ where core levels of (A) $\mathrm{Cl}$, (B) O1s and (C) N1s and (D) S $2 p_{3 / 2}$ and S $2 p_{1 / 2}$ are deconvoluted separately. The circles represent the experimental data; thick lines represent the fitting data for overall signal; the dotted lines are the deconvoluted individual peaks for different species present in the sample.

sponding to various groups on the sidewalls generated during the synthesis. Figure 5a $(01 \mathrm{~s})$ shows five different peaks after a similar fitting procedure; a peak at a binding energy of $529.5 \mathrm{eV}$ corresponds to the oxygen in $-\mathrm{SO}_{3} \mathrm{H}$ group, while intense peaks at $531.2 \mathrm{eV}$ and $532.4 \mathrm{eV}$ could be attributed to the double bonded oxygenated group like $-\mathrm{COOH}$ and group like $-\mathrm{NO}_{2}$ respectively. Similarly, a peak at $533.2 \mathrm{eV}$ could be assigned to the single bonded oxygenated groups like $-\mathrm{OH}$ whereas, a smaller peak at $534.3 \mathrm{eV}$ might be due to adsorbed moisture or oxygen. In addition, a broad and less intense peak observed at $399.8 \mathrm{eV}$ (figure $5 \mathrm{a}(\mathrm{Nls})$ ) is assigned to the $\mathrm{N}$ 1s core level of $-\mathrm{NO}_{2}$ group. A doublet at 166.9 and $168.0 \mathrm{eV}$ (figure 5a; $\mathrm{S} \mathrm{2p}$ ) has been assigned to the $\mathrm{S} 2 p_{3 / 2}$ and $\mathrm{S} 2 p_{1 / 2}$ level of $-\mathrm{SO}_{3} \mathrm{H}$ groups respectively, which are developed during treatment.

Figure 5b illustrates the XP spectra of SWNTs after covalent bonding with tridecylamine due to several environmental changes. Interestingly, the $\mathrm{Cls}$ peak is found to be affected after amidation, which is evidenced in figure $5 \mathrm{~b}(\mathrm{Cls})$, where the peak $(284.5 \mathrm{eV})$ due to graphitic $\mathrm{C}-\mathrm{C}$ bonding is reduced appreciably and a new peak appeared at $286.7 \mathrm{eV}$ could be attributed to $\mathrm{Cls}$ of alkyl chain of amine. This enhanced and unusual intense peak (intensity: 82820; FWHM: 2.02) corresponding to alkyl carbon indicates a remarkable degree of amidation. In addition, two peaks at 287.9 and $290.1 \mathrm{eV}$ are assigned to $\mathrm{C}-\mathrm{OH}$ and $-\mathrm{CONH}$ linkages, although a slight shift is observed in both the cases that might be due to several complex interactions with other functional groups. On normalizing with respect to $\mathrm{N}$, the atomic ratio $\mathrm{C}: \mathrm{N}$ of $8.6: 1(6.8 \mathrm{wt} \%$ of $\mathrm{N}$ with respect to total C) is observed which is in an excellent agreement with that of $\mathrm{C}$ : $\mathrm{COOH}$ of s-SWNTs. This ratio is also verified by comparing the $\mathrm{C} 1 s(290 \cdot 1 \mathrm{eV})$ and N1s $(401.8 \mathrm{eV})$ of amide linkages, which is found to be $1: 1$. Also, an atomic ratio of $16: 1$ for $\mathrm{N}: \mathrm{S}$ (and $\mathrm{C}: \mathrm{S}$ as $175: 1$ i.e. $0.76 \mathrm{wt} \%$ of $\mathrm{S}$ ) has been observed, indicating a lesser extent of $-\mathrm{SO}_{3} \mathrm{H}$ groups developed during microwave treatment. Further, appreciable changes have been observed in case of $\mathrm{O} 1 \mathrm{~s}$ peaks after amide formation. Figure $5 \mathrm{~b}$ (O1s) indicates the deconvoluted spectra for $\mathrm{Ols}$, which shows four peaks corresponding to $-\mathrm{SO}_{3} \mathrm{H}$, $-\mathrm{OH},-\mathrm{NO}_{2}$, and $-\mathrm{CONH}$ bonding at $531 \cdot 1,532 \cdot 0$ and 533.1 and $533.45 \mathrm{eV}$ respectively. Minor contribution from adsorbed oxygen is not observed even after fitting up to standard deviation $\left(\chi^{2}\right)$ of less than unity. Also, an extra peak at $401.8 \mathrm{eV}$ (intensity: 10716; FWHM: 2.06) observed in case of N1s might be due to - $\mathrm{CONH}-$ linkages, which is shown in figure $5 \mathrm{~b}(\mathrm{~N} 1 \mathrm{~s})$. Interestingly, two minor peaks observed at 176.5 and $177.5 \mathrm{eV}$ in case of $\mathrm{S} 2 p_{3 / 2}$ after amidation, could be due to $-\mathrm{SO}^{-}$moieties attached to halide like chloride ions or due to the formation of salt like SWNTs-SO ${ }_{3}{ }^{\oplus} \mathrm{NH}_{2}\left(\mathrm{CH}_{2}\right)_{12} \mathrm{CH}_{3}$ (figure $4 \mathrm{~b}$ (S2p)). Consequently, a remarkable solubility ranging from 2.6 to $5 \mathrm{mg} / \mathrm{mL}$ is observed after MW treatment, which could be due to a higher concentra- 
tion $(\sim 30 \%)$ of oxygenated species including $-\mathrm{COOH},-\mathrm{OH},-\mathrm{SO}_{3} \mathrm{H}$ and $-\mathrm{NO}_{2}$ groups developed during microwave irradiation.

Raman spectroscopy has also been employed to strengthen our interpretation of clean and efficient functionalization. Accordingly, figure 6a shows superimposed Raman spectra of pristine-SWNTs (A), s-SWNTs (B) and tridecylamine derivatized SWNTs (C). Shifts in the D (disorder or defect sites in the sample) and G-bands (tangential mode) after the microwave treatment and eventually by tridecylamine functionalization, clearly suggest an enormous side wall damage resulting into effective functionalization of $s p^{2}$ carbon. The microwave treatment on SWNTs in presence of such concentrated acid mixture gives a remarkable degree of solubilization and debundling, facilitating the narrow distribution of nanotube diameter. Interestingly, this has been found to be less feasible in case of single acid treatment. Most importantly, an increase in the ratio $I_{G} / I_{D}$ observed in the case of s-SWNTs appears to contra-

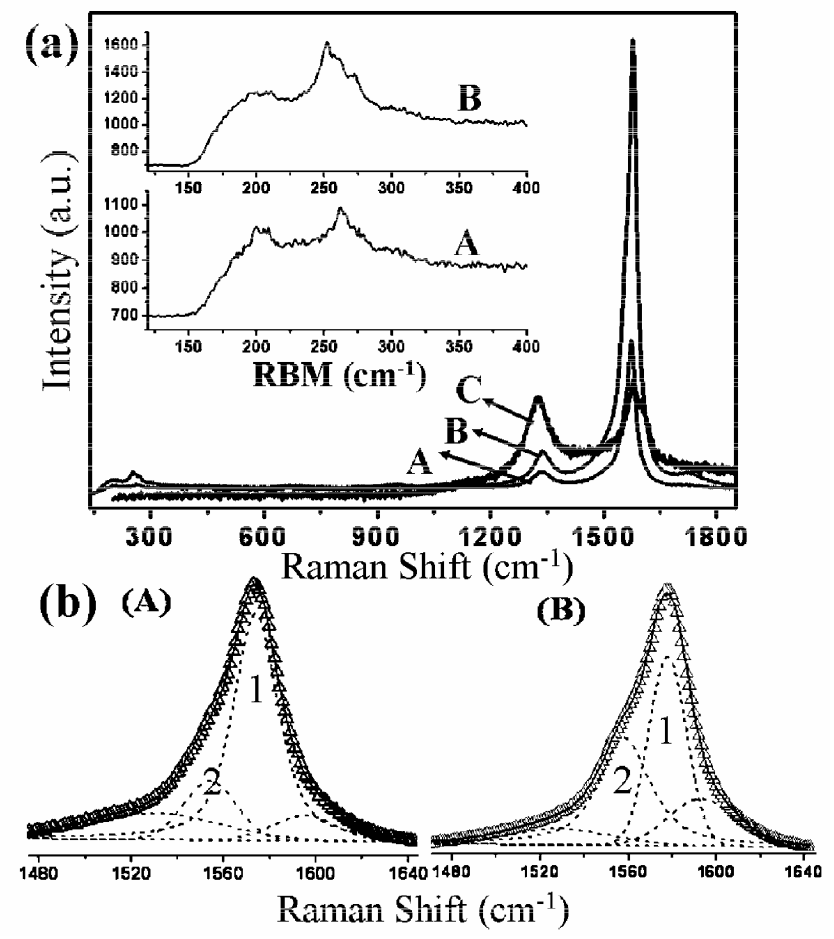

Figure 6. (a) Superimposed Raman spectra of (A) pristine-SWNTs, (B) s-SWNTs and (C) SWNTs-CONH $\left(\mathrm{CH}_{2}\right)_{12} \mathrm{CH}_{3}$, which indicates a relative decrease in $I_{\mathrm{G}} / I_{\mathrm{D}}$ ratio revealing amine functionalization, (b) G-band splitting of (A) pristine-SWNTs and (B) s-SWNTs revealing an increase in the peak at $1558 \mathrm{~cm}^{-1}$ causing much damage to the side walls after microwave treatment; inset of (a) shows RBM region of pristine-SWNTs and s-SWNTs. dict the findings of previous reports, where a chemical functionalization adds to a defect site contribution. ${ }^{25,26}$ This is quite acceptable in the present case, since removal of non-graphitic carbon impurities and catalyst particles after MW treatment can reduce the D-band intensity, and hence logically, such treatment would cause an additional burden to the purity of sample. Further, the RBM peaks are absent in case of SWNTs-CONH $\left(\mathrm{CH}_{2}\right)_{12} \mathrm{CH}_{3}$, perhaps due to strong covalent wrapping of tridecylamine on the nanotubes (clearly evidenced in the TEM images, figure lc-d). Significantly, the G-band has been affected seriously, shifting to higher wavenumbers by $4 \mathrm{~cm}^{-1}$, which can further be clarified by looking at the splitting after the microwave treatment. The deconvolution of this G-band gives a decrease of peak ratio (marked as 1 and 2 in figure $6 \mathrm{~b}$ ) suggesting a higher degree of sidewall functionalization or debundling of SWNTs leading to the defect site formation, which could further facilitate the amine functionalization. In addition, figure $6 \mathrm{a}$ (C) shows a Raman spectrum of tridecylamine functionalized SWNTs, revealing a decreased $I_{G} / I_{D}$ ratio presumably indicating a remarkable extent of amide linkages on the side walls as well as on the broken ends.

Except the side wall functionalization, the rest of the nanotube network and its electronic structure have been preserved during such functionalization, which is evidenced by DRIFT and Raman spectroscopy. In addition, the unusual solubility of both sSWNTs and SWNTs-CONH $\left(\mathrm{CH}_{2}\right)_{12} \mathrm{CH}_{3}$ in their respective solvents might be due to the covalent functionalization rather than non-covalent. ${ }^{26-28}$

\section{Conclusion}

This novel technique for the complete phase transformation of SWNTs/MWNTs from aqueous to organic medium could pave the way for a new generation of CNT composites capable of controlling their unique properties in a determinable manner. A maximum of $30 \mathrm{wt} \%$ of oxygenated groups have been generated on the side walls by rapid MW treatment, leading to a solubility of $2.6 \mathrm{mg} / \mathrm{mL}$ in water. A clean and higher, yet quantitative, degree of amidation is achieved by using oxalyl chloride preceded by microwave treatment in acid media. UV-Vis analysis showed the disappearance of singularities after MW treatment and covalent amidation with tridecylamine, indicating the blockage for valence electrons due to functionalization. ${ }^{1} \mathrm{H}$ NMR 
and Raman spectroscopic studies also showed the features that supports for a covalent functionalization of SWNTs with tridecylamine. The practical utilization of such tunable CNT surfaces, both in aqueous as well as in non-aqueous media, to make high performance polymer composites (e.g. controlling the interfacial adhesion between CNT surface and polymer) would significantly open up new perspectives in the preparation of various polymer composites extending the range of their possible applications.

\section{Acknowledgements}

University Grants Commission (UGC) and Council for Scientific and Industrial Research (CSIR) through NMITLI project for the financial support. We thank Dr Sivaram, Director, National Chemical Laboratory (NCL) for his constant encouragement.

\section{References}

1. Salvetat J P, Bonard J M, Thomson N H, Kulik A J, Forro L, Benoit W and Zuppiroli L 1999 Appl. Phys. A: Mater. Sci. Process 69255

2. Calvert P 1999 Nature 399210

3. Dai H 2002 Acc. Chem. Res. 351035

4. Pantarotto D, Partidos C D, Hoebeke J, Brown F, Kramer E, Briand J P, Muller S, Prato M and Bianco A 2003 Chem. Biol. 10961

5. Chen R J, Zhang Y, Wang D and Dai H J $2001 \mathrm{~J}$. Am. Chem. Soc. 1233838

6. Strano P J, Dyke C A, Usrey M L, Barone P W, Allen M J, Shan H, Kittrell C, Hauge R H, Tour J M and Smalley R E 1999 Science 3011519

7. Liu J, Rinzler A G, Dai H, Hafner J H, Bradley R K, Boul P J, Lu A, Lverson T, Shelimov K, Huffman C B, Rodriguez-Macias F, Shon Y S, Lee T R, Colbert D T and Smalley R E 1998 Science 2801253

8. Bahr J L and Tour J M 2002 J. Mater. Chem. 121952

9. Banerjee S, Kahn M G C and Wong S S 2003 Chem. Eur. J. 91898
10. Liu T and Kumar S 2003 Nano Lett. 5647

11. Liang F, Sadana A K, Peera A, Chattopadhyay J, Gu Z, Hauge R H and Billups W E 2004 Nano Lett. 4 1257

12. Zhao W, Song C and Pehrsson P E $2002 \mathrm{~J}$. Am. Chem. Soc. 12412418

13. Qin S, Qin D, Ford W T, Herrera J E, Resasco D E, Bachilo S M and Weisman R B 2004 Macromolecules 373965

14. Zhao B, Hu H and Haddon R C 2004 Adv. Funct. Mater. 1471

15. Georgakilas V, Tagmatarchis N, Pantarotto D, Bianco A, Briand J P and Prato M 2002 Chem. Commun. 24 3050

16. Pompeo F and Resasco D E 2002 Nano Lett. 2369

17. Takahashi T, Tsunoda K, Yajima H and Ishii T 2002 Chem. Lett. 7690

18. Davis V A, Ericson L M, A N Parra-Vasquez, H Fan, Y Wang, V Prieto, J A Longoria, S Ramesh, R K Saini, C Kittrell, W E Billups, W W Adams, R H Hauge, Smalley R E and Pasquali M 2004 Macromolecule 37154

19. Hudson J L, Casavant M J and Tour J M $2004 \mathrm{~J}$. Am. Chem. Soc. 12611158

20. Wang Y, Iqbal Z and Mitra S $2006 \mathrm{~J}$. Am. Chem. Soc. 12895

21. Saini R K, Chiang I W, Peng H, Smalley R E, Billups W E, Hauge R H and Margrave J L $2003 \mathrm{~J}$. Am. Chem. Soc. 1253617

22. Hamon $\mathrm{M} \mathrm{A}, \mathrm{Hu} \mathrm{H}$, Bhowmik $\mathrm{P}$, Niyogi S, Zhao B, Itkis M E and Haddon R C 2001 Chem. Phys. Lett. 3478

23. Mark H, Chen J, Hu H, Chen Y, Itkis M E, Rao A M, Eklund P C and Haddon R C 1999 Adv. Mater. 11 834

24. Chen J, Hamon $\mathrm{M} \mathrm{A}, \mathrm{Hu} H$, Chen $\mathrm{Y}$, Rao A M, Eklund P C and Haddon R C 1998 Science 282 95

25. Gu Z, Peng H, Hauge R H, Smalley R E and Margrave J L 2002 Nano Lett. 21009

26. Banerjee S, Benny T H, Balasubramanian M, Fischer D A, Misewich J A and Wong S S 2004 Chem. Phys. Chem. 51416

27. Hentschke R and Winkler R G 1993 J. Chem. Phys. 995528

28. Park C, Engel A, Crowe E S and Rodriguez N M 2000 Langmuir 168050 